\title{
A SIMPLE APPROACH TO UNDERSTAND SOLID STATE CHEMISTRY WITH THE EXAMPLE OF A PIEZOELECTRIC MATERIAL INCORPORATED IN A BIRTHDAY CARD SPEAKER
}

\author{
A. Carretero-Cerdán, A. Peiró-Franch, R. Torres-Iglesias, L. Trigueros-Navarro, \\ E. Cordoncillo*, H. Beltrán-Mir \\ Departament de Química Inorgànica i Orgànica. Universitat Jaume I, Avda. Sos Baynat s/n, \\ Castelló (SPAIN) \\ Students from "Máster en Química Aplicada y Farmacológica \\ (Intensificación en Materiales Avanzados)" \\ *Lecturers of the "Máster en Química Aplicada y Farmacológica"
}

\begin{abstract}
Advanced solid materials are present in a large quantity of fields of our quotidian live. All the electronic devices surrounding us, such as mobile phones, computers or appliances are made up of many different materials: ceramics, metals, polymers, etc. The recent development of advanced ceramic materials to be applied in electronic devices, which require high performances as well as relatively economic syntheses and environmentally friendly characteristics, is a wide field in Materials Chemistry. It is important to motivate students to this area, so that, they can understand the importance of the chemistry and the material science in our society. In the present contribution, we would like to relate our experience giving a comprehensive laboratory session intended for all undergraduate chemistry students.
\end{abstract}

Piezoelectric compounds can be defined as materials that produce some electric charge when a mechanical stress is applied (direct effect) or suffer a mechanical deformation when an electric field is applied (reverse effect). These materials are used in several devices such as lighters, speakers, sensors or sonars. Lead zirconate titanate $\left[\mathrm{Pb}\left(\mathrm{Ti}_{1-x}, \mathrm{Zr}_{x}\right) \mathrm{O}_{3}\right](\mathrm{PZT})$ is the most studied and commercialized ceramic piezoelectric material due to their excellent characteristics. Relation between structure-composition-properties of this material and its easy preparation makes it suitable to use in low value added devices.

In this work, we propose an example to understanding this technology based on the components of a speaker of a musical birthday card, in particular the piezoelectric component, including the chemistry that is involved in these types of devices. Two common characterization techniques in Solid State Chemistry are also introduced to the students: X-Ray Diffraction and Electron Microscopy. Moreover, the discovery by the students of the presence of this type of material in this simple device offers a possibility to motivate them to learn these sciences, and allow them to know the importance of the Solid State Chemistry and the Materials Science in our society.

Different educational methodologies may be used to study and understand these materials with piezoelectric properties. Problem Based Learning (PBL) is a widely applied approach intended to encourage students to learn through the structured exploration of a research problem. Currently this particular system has become a valid teaching method in high schools, where students are encouraged to develop a real research project. The PBL methodology used in this work represents an improvement in the content of the knowledge while simultaneously it fosters the development of communication, problem-solving, and self-directed learning skills.

This work can also be interesting for teachers of inorganic chemistry and material science and undergraduate students owing to its pedagogical character.

Keywords: Problem Based Learning, piezoelectric materials, Solid State Chemistry, Material Science.

\section{INTRODUCTION}

Materials have played a very important role in the History of Humanity making life easier. Evolution has always been linked to developments in materials: starting with the stones used to make fire, one of the most important progresses of Humanity. Metallurgy and ceramic, both developed in the 
Prehistory, were also great advances. In fact, the Periods of Prehistory were named after the materials that were being developed, e.g. Stone Age, Bronze Age, Iron Age.

In the recent years, there has been a breakthrough in technology that has made possible the improvement of already known materials and the creation of new ones. For example, the miniaturization of devices has become a dominant technological development, especially in electronic devices.

A surprisingly large proportion of chemistry graduates at all levels take jobs in industries specializing in phosphors, lasers, and other ceramic, electronic, nuclear, magnetic and optical materials. Indeed, today more and more chemists are accepting jobs in these applied areas. Many of these materials are based on oxide systems and must be prepared at high temperatures and characterized nondestructively in the solid state. Although the chemistry departments of a few universities have exemplar training in high temperature and Solid State Chemistry at the graduate level, most have none at either the undergraduate or graduate level.

Solid State Chemistry is a wide field in Chemistry which studies the synthesis, structure, properties and characterization of solid materials. It has a strong overlap with solid state physics, mineralogy, crystallography, ceramics, metallurgy, thermodynamics, materials science and electronics. Nowadays, its main objective is the development of advanced materials with improved features such as optical, electrical, magnetic or mechanical properties. For example, these materials range from the pieces that built a phone to artificial teeth.

Different educational methodologies may be used to study and understand these materials with piezoelectric properties. Problem-Based Learning (PBL) is a very useful tool employed for the first time at McMaster University, in Canada. It is based on the idea that the student seeks the solution of a real problem. This methodology allows students to take responsibility for their own learning, work in group and improve their communication and creativity. For this reason, PBL is a technique used by teachers and lecturers in their courses at many areas of study [1-4]. This method could be used to get a better understanding of materials present in different objects of our quotidian life. To apply this tool, in this work we propose an example to understanding the components in a speaker of a musical birthday card, in particular the piezoelectric component, including the chemistry that is involved in these types of devices.

Therefore, the main aim of this work is the study of a simple illustrative example of a piezoelectric material present in a real device. Materials with piezoelectric properties are present in many everyday objects, such as lighters, cameras, headphones, sonograms, speakers, wristwatches, etc. We introduced the solid state characterization techniques to the students through a birthday card speaker. These devices contain a material with a piezoelectric effect. The birthday card had an acoustic part, the piezoelectric material, and a light part, a LED (Light-Emitting Diode), Fig. 1. We are going to focus in the acoustic part and the fundamentals of the basic materials present in this component are described below. Two common characterization techniques in Solid State chemistry are also introduced to the students: X-Ray Diffraction and Scanning Electron Microscopy. Fundamentals of these techniques are also explained later.

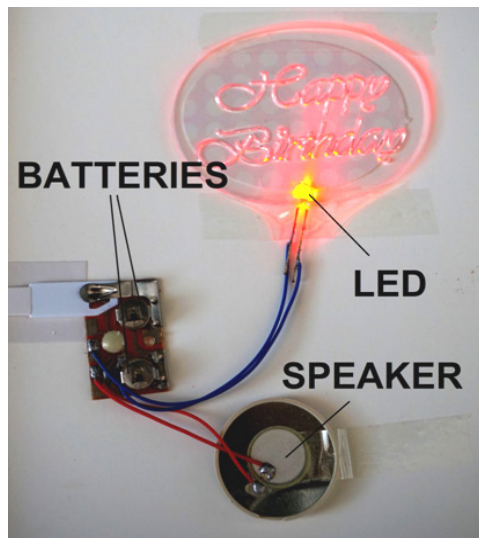

Figure 1. Components of the birthday card.

This work encourages students to learn through the structured exploration of a research problem and allows them to have a better understanding of Solid State Chemistry. This work can also be interesting 
for general public, teachers of inorganic chemistry and materials science and undergraduate students owing to its pedagogical character.

\section{FUNDAMENTALS OF PIEZOELECTRIC MATERIALS}

In this section, the fundamentals of the piezoelectric materials are described as sequential steps. Sequential learners tend to gain understanding in linear steps, with each step following logically from the previous one, and tend to follow logical stepwise paths in finding solutions. Students might also try to strengthen their thinking skills by relating each new topic they study to things they already know. The more they can do so, the deeper their understanding of the topic is likely to be. Therefore, each concept in this section will be separately indicated, showing the relation between composition, structure and properties of these kind of materials.

\section{+ First, students have to define what a piezoelectric material is.}

Piezoelectricity was observed for the first time at the beginning of the 18th century in a tourmaline gemstone brought from the East Indies by the Dutch. This stone had a peculiarity, when it was being heated, it could attract other materials, like ashes. This phenomenon was explained in 1880 by the brothers Pierre and Jacques Curie, who observed that when some crystals, such as tourmaline, quartz or topaz, were brought under a mechanical stress, electricity was produced. To describe this effect, they combined the observed properties with the already known pyroelectric effect. The pyroelectric effect arises because the thermal expansion that occurs on heating changes the size of the intrinsic dipoles. The following year, Gabriel Lippman demonstrated mathematically that the reverse effect could also occur, and the Curie brothers proved it experimentally. These behaviours were named as piezoelectric from the Greek word piezein, which means press.

Piezoelectricity is a property present in some crystalline materials that consists in their polarization, and consequently, the appearance of a voltage, once those materials are brought under mechanical stress. This is known as the direct piezoelectric effect. The reverse effect may also occur, i.e. when an electric field is applied, the material is deformed. This effect is reversible and depends on the crystal structure and direction of the applied stress. Direct and reverse piezoelectric effect are intrinsic in some materials (e.g. sugar, quartz, bone), but it can also be induced by using a magnetic field [5]. Fig. 2 summarizes the piezoelectric effect.

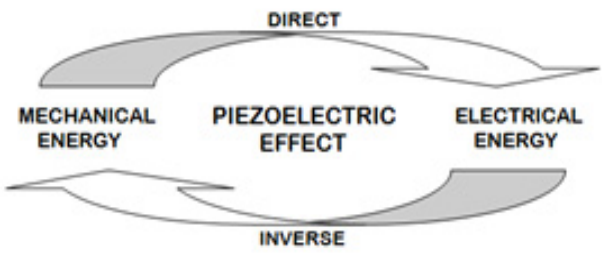

Figure 2. Scheme of the piezoelectric effect.

+ Second, students have to identify the composition and structure of the most used piezoelectric material.

The most known piezoelectric material, PZT (lead zirconate titanate, $\left[\mathrm{Pb}\left(\mathrm{Ti}_{1-\mathrm{x}} \mathrm{Zr}_{\mathrm{x}}\right) \mathrm{O}_{3}\right]$ ), was discovered in 1952. It is a ceramic material with a perovskite structure, $A^{I I} B^{I V} O_{3}$, shown in Fig. 3 . In the perovskite structure, $\mathrm{A}$ is a large cation (alkali metal, alkaline earth metal or lanthanide), B is a medium-sized cation (transition metal) and $X$ is generally oxygen or one of the chalcogenides ( $\mathrm{S}, \mathrm{Se}$ or $\mathrm{Te}$ ). The divalent cation, $A$, occupies the vertices of the unit cell, with a coordination number $(C N)$ of 12 ; the tetravalent cation, $\mathrm{B}$, is in an octahedral hole in the center of the unit cell, $\mathrm{CN}=6$; and $\mathrm{X}$ is in the center of the faces, with $\mathrm{CN}=6$. In particular, in the PZT material the elements are: $\mathrm{A}=\mathrm{Pb}(\mathrm{II}), \mathrm{B}=\mathrm{Ti}(\mathrm{IV})$ and $\mathrm{Zr}(\mathrm{IV})$, and $\mathrm{X}$ is oxygen. $\mathrm{PZT}$ is a solid solution where some titanium ions are substituted by zirconium, $\left[\mathrm{Pb}\left(\mathrm{Ti}_{1-\mathrm{x}} \mathrm{Zr}_{\mathrm{x}}\right) \mathrm{O}_{3}\right]$. Depending on the value of $\mathrm{x}$, i.e. the proportion of $\mathrm{Ti}$ and $\mathrm{Zr}$, the structure of the material is different and also the properties. The Curie point is the temperature in which there is structural change. Above the Curie point, the structure of PZT is cubic, symmetric and it does not show piezoelectricity (Fig. 3a). If the temperature decreases below that point (Fig. 3b), there is a change in the structure: it goes from cubic to tetragonal or rhombohedral and it loses its symmetry, which leads to create an unequal distribution of charge inside the crystal, and it becomes piezoelectric 
[6]. The application of an electric field yields the distribution of these charges and the generation of local electric dipoles within the material, which are responsible of the piezoelectric effect.

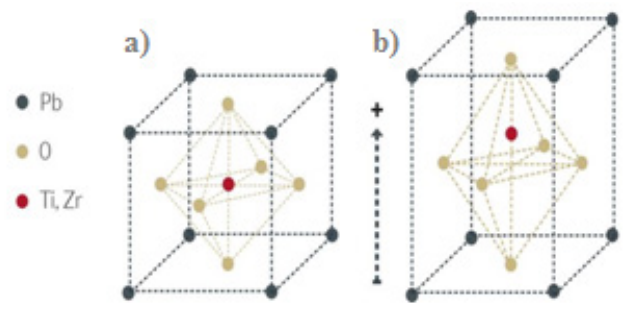

Figure 3. Perovskite structure of PZT above (a) and below (b) the Curie point.

+ Third, once the structure is identified, students are introduced in the field of the equilibrium phase diagrams. Therefore, in this case, they analyze the phase diagram of the system $\mathrm{PbTiO}_{3}-\mathrm{PbZrO}_{3}$.

Perovskites in the system $\mathrm{PbTiO}_{3}-\mathrm{PbZrO}_{3}$ have been extensively studied. They show superior piezoelectric properties in combination with a relatively high Curie temperature. The phase diagram of $\mathrm{PbTiO}_{3}-\mathrm{PbZrO}_{3}$ system is shown in Fig. 4. This phase diagram represents the crystal structure in which a material crystallizes at a certain temperature and composition. In this particular diagram, the boundary between tetragonal and rhombohedral crystal structures is nearly just depending on temperature and it is known as the morphotropic phase boundary (MPB). The best piezoelectric materials are found in a range near to a 1:1 composition of $\mathrm{PbTiO}_{3}: \mathrm{PbZrO}_{3}$, which is close to the MPB.

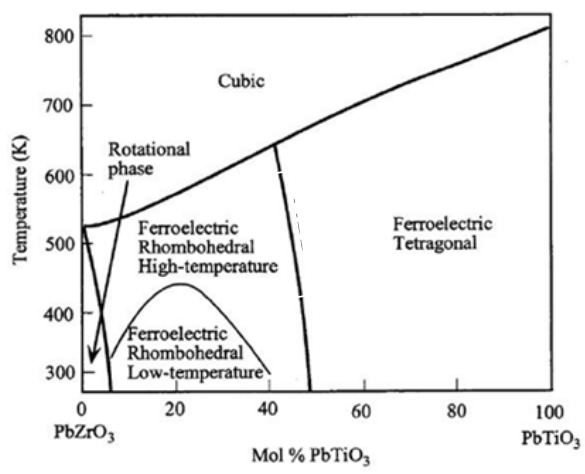

Figure 4. Phase diagram $\mathrm{PbTiO}_{3}-\mathrm{PbZrO}_{3}$ system [7].

+ Fourth, at this point, students discuss the environmental problems of this system, in particular the toxicity of lead.

The main problem that PZT presents is its lead content, a toxic element, banned in many countries. Lead is known worldwide due to its human applications: it can be found in batteries and accumulators, ammunition, old pipes, old paints or car fuel. This widespread use has caused significant pollution and affected the human health, because it accumulates in living organisms and can enter into the body through the digestive or respiratory tract, causing serious diseases [8]. To carry out this work, the necessary precautions have been taken, avoiding any risk to health.

Alternative non-lead materials to PZT and its derivatives have been developed in recent years, although, so far, lead-free piezoelectric ceramics with the excellent properties of PZT have not been synthesized yet. An example of a lead-free piezoelectric material is $\mathrm{K}_{0.5} \mathrm{Na}_{0.5} \mathrm{NbO}_{3}(\mathrm{KNN})$, mixed with elements like lithium, tantalum, among others [9].

+ Finally, students analyze how these materials are presented in a device and their performance.

One of the main applications of these materials is in lighters as the generator of the electric spark when it is subjected under mechanical stress (direct effect) and in speakers, where electric impulses are applied and the deformation of the piezoelectric material generates the sound (reverse effect). When voltage is applied the constituents in the crystal are subjected to electrical pressure and it is why the material is mechanically stressed (deformed in shape). These deformations are shown in Fig. 5. 
There are many other applications such as ultrasonic transducers, actuators, vibration sensors, capacitor arrays, electronic- caloric coolers for computers, etc.

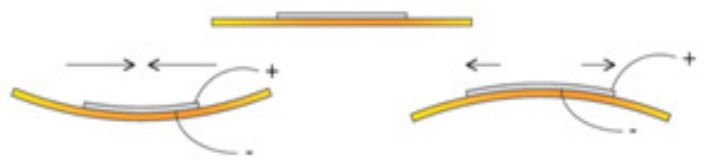

Figure 5. Deformation of the piezoelectric material in the speaker when voltage is applied.

\section{CHARACTERIZATION TECHNIQUES}

One of the aims of this work is to introduce students the two most common techniques used in Solid State Chemistry and show them the potential of these techniques to characterize solid materials.

In order to characterize the different components of the birthday card, and in particular of the piezoelectric material, two of the most important techniques in Solid State Chemistry were used: XRay Diffraction and Scanning Electron Microscopy (SEM) with a spectrometer for energy dispersive analysis of X-ray (EDX). The main characteristics of these techniques are explained below.

\subsection{X-Ray Diffraction (XRD)}

The XRD technique is commonly used to identify the crystalline phases of an inorganic solid. X-rays are electromagnetic radiation of a wavelength of $10 \AA\left(10^{-10} \mathrm{~m}\right)$. They occur in the part of the electromagnetic spectrum between $\mathrm{Y}$-rays and the ultraviolet. $\mathrm{X}$-rays are produced when high-energy charged particles (e.g. accelerated electrons) collide with matter. The electrons are slowed down or stopped by the collision and some of their lost energy is converted into electromagnetic radiation. The $\mathrm{X}$-rays which are used in almost all diffraction experiments are produced by a process that leads to monochromatic X-rays, where a beam of accelerated electrons strike a metal target, usually copper. The incident electrons have sufficient energy to ionize some of the copper electrons. In consequence of this ionization, an electron in an outer orbital immediately drops down to occupy the created vacancy and the energy released in the transition appears as X-radiation.

Crystals have regularly repeating structures and are capable of diffracting radiation with a wavelength similar to the interatomic separation, such as the X-ray radiation. The Bragg approach to diffraction is to regard crystals as built up in layers or planes such that each acts as a semi-transparent mirror. Some of the X-rays are reflected off a plane with the angle of reflection equal to the angle of incidence, but the rest are transmitted to be subsequently reflected by succeeding planes. These reflections are capable to combine constructively or destructively depending on the interplanar distance (d). The condition for constructive interference to occur is:

$$
\mathrm{n} \lambda=2 \mathrm{~d} \sin \theta
$$

This is known as Bragg's Law for X-ray diffraction, where the path difference between two waves undergoing constructive interference is given by $2 d \sin \theta, \theta$ is the scattering angle, $n$ is an integer number and $\lambda$ is the wavelength applied in the sample.

When Bragg's Law is satisfied, the reflected beams are in phase and interfere constructively. These interferences can be represented in a diffractogram, which is characteristic for each compound and can be used to identify, principally, inorganic systems in Solid State Chemistry. At angles of incidence other than the Bragg angle $(\theta)$, reflected beams are out of phase and destructive interference or cancellation occurs [10].

Today, the entire well known systems and the new developed compounds are identified by $\mathrm{X}$-ray diffraction and there is a database called JCPDS-ICDD (Journal Committee Powder Diffraction Standard-International Center of Diffraction Data) that contains their diffractograms. If a diffractogram of a new material is stored in this database, its characterization can be done.

Phase analysis was performed by X-ray powder diffraction (XRD) with a Bruker AXS D4 Endeavor diffractometer with CuKa radiation. Data were collected by step scanning over a $2 \theta$ range from $10^{\circ}$ to $70^{\circ}$ with a step size of $0.05^{\circ}$ and $1.5 \mathrm{~s}$ counting time at each step. The goniometer was controlled by the "DIFFRACT plus" software, which also determined diffraction peak positions and intensities. The instrument was calibrated using an external Si standard. 


\subsection{Scanning Electron Microscopy (SEM)}

A scanning electron microscopy (SEM) provides morphological and topographic information about the surface of the solid samples by scanning them with a high-energy beam of electrons in a raster scan pattern. The electrons interact with the atoms that make up the sample producing signals that contain information about the sample's surface topography, composition, and other properties such as electrical conductivity [11].

In SEM instruments, backscattered and secondary electrons are detected and used to construct the image. In the image obtained from backscattered electrons, it is possible to observe a qualitative mapping of the elements by the different atomic weight; the heavier the element, the brighter this area. Therefore, a SEM is an instrument (powerful microscopy) that permits obtaining high-resolution images. For chemical analysis purposes, SEMs also have X-ray detectors that allow semiquantitative determinations.

Scanning electron micrographs of the samples were taken on a SEM JEOL 7001F model, equipped with a spectrometer for energy dispersive analysis of X-rays (EDX). The samples for microstructure determination and microanalysis were deposited on an Al holder coated with platinum. Scanning electron microscopy provides information about the microstructure and therefore, the sintering of the material, whereas EDX give information about the composition.

\section{THE COMPONENTS OF THE BIRTHDAY CARD}

The first aim of the work is showing students the relationship between structure and properties of a solid material. For this purpose, the components of the birthday card were analyzed, in particular the piezoelectric material.

The analysis of the components of the birthday card speaker were carried out in order to understand how it works and to study the features of the piezoelectric material. The card was disassembled and its setup is shown in Fig. 6. It consists of (a) two batteries, (b) a Light Emission Diode (LED), and (c) the speaker containing the piezoelectric material. The speaker is covered with a plastic, which plays the role of an acoustic box, and it also has two metallic contacts.

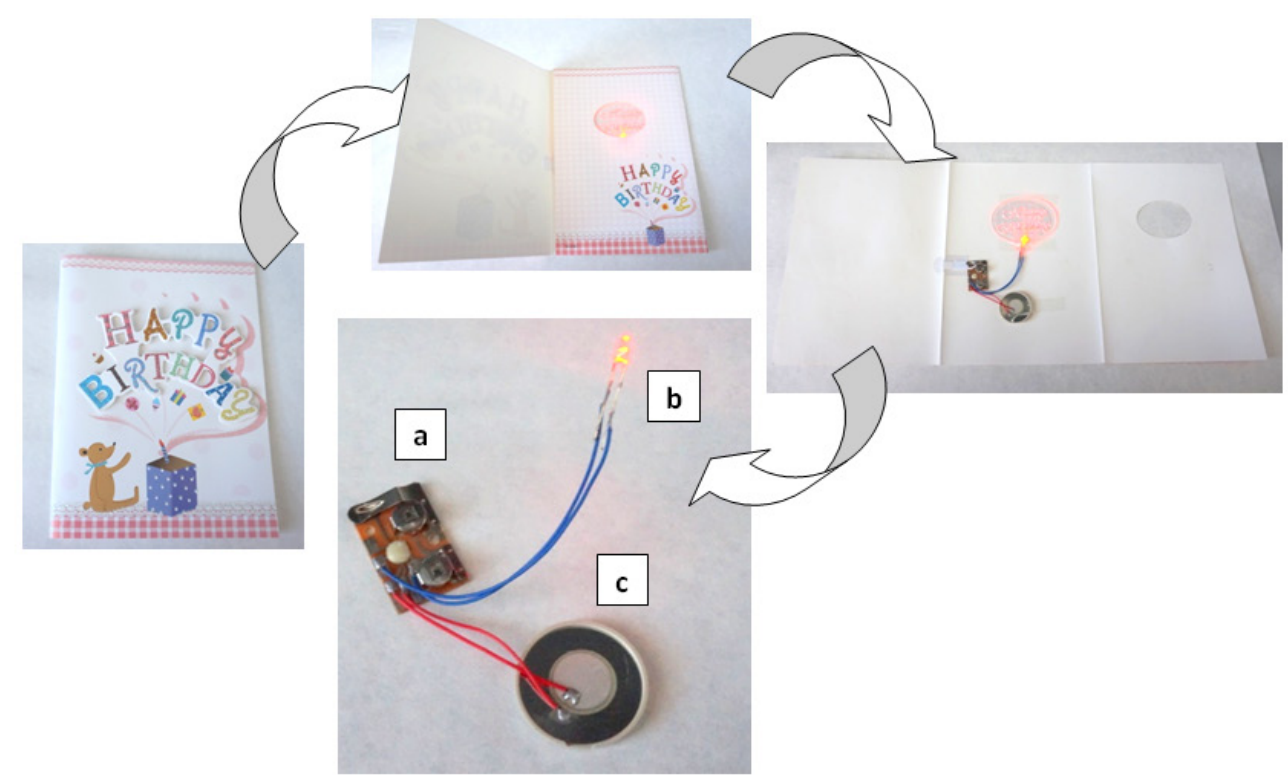

Figure 6. Disassembled birthday card: (a) batteries, (b) LED, (c) speaker.

\section{CHARACTERIZATION OF THE MATERIALS IN THE SPEAKER}

Results obtained by using the techniques mentioned above are explained in this section, following a logical PBL. 
Fig. 7 shows the setup of the speaker. At naked eye, three layers are observed: two metallic electrodes (layers I and III) and the piezoelectric material (layer II). Each constituent of this device was separately analyzed.

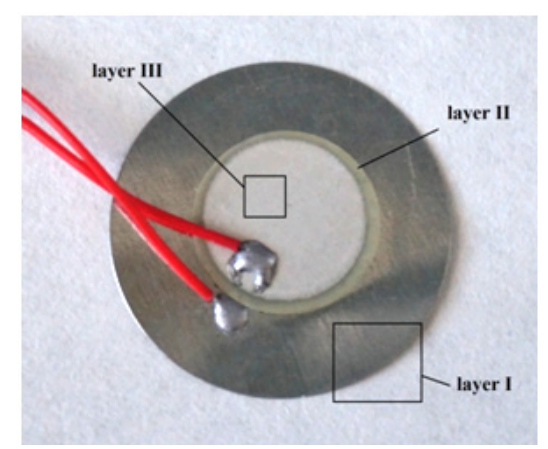

Figure 7. Setup of the speaker.

Prior to the analysis of the layers, students should know that a piezoelectric material must be coated on both sides by conductive materials acting as the electrodes.

Layer I was analyzed by SEM/EDX and metallic tin was detected. Then, layer II was also analyzed by SEM/EDX. Fig. 8(a) shows a SEM image of this layer, which consists of two distinct regions: the white region whose microstructure corresponds to a ceramic material, and the black one which seems to be a polymeric material. These regions were separately studied and compositional microanalyses were obtained by EDX.

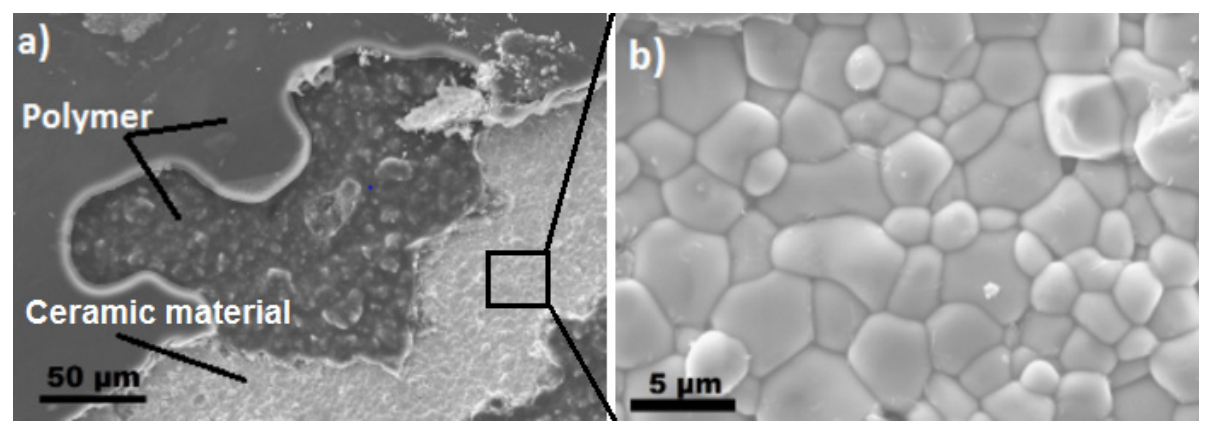

Figure 8. (a) SEM image of Layer II, (b) SEM image of the ceramic material at higher magnification.

Table 1. EDX analysis of the ceramic material.

\begin{tabular}{|c|c|}
\hline Element & Atomic percent \\
\hline $\mathrm{Ti}$ & 8.25 \\
\hline $\mathrm{Zr}$ & 10.76 \\
\hline $\mathrm{Pb}$ & 19.92 \\
\hline $\mathrm{O}$ & 61.06 \\
\hline
\end{tabular}

The EDX analysis of the black region showed a characteristic elements of an organic material with a great content of carbon and oxygen. This organic material could be identified as a conductive polymer. This polymer may work as junction agent.

In order for students to understand the microstructure of the ceramic material, a micrograph of the white region was performed at higher magnification, Fig. 8 (b). The ceramic was formed by irregular grains with particle sizes between 2 and $5 \mu \mathrm{m}$. EDX of this region showed the composition described in Table 1, with atomic ratios of $\mathrm{Pb}: \mathrm{Zr}: \mathrm{Ti} 1: 0.54: 0.41$, giving a stoichiometry close to $\mathrm{Pb}\left(\mathrm{Zr}_{0.54}, \mathrm{Ti}_{0.41}\right) \mathrm{O}_{3.06}$. This composition is characteristic of the most common piezoelectric material, $\mathrm{Pb}(\mathrm{Zr}, \mathrm{Ti}) \mathrm{O}_{3}(\mathrm{PZT})$. 


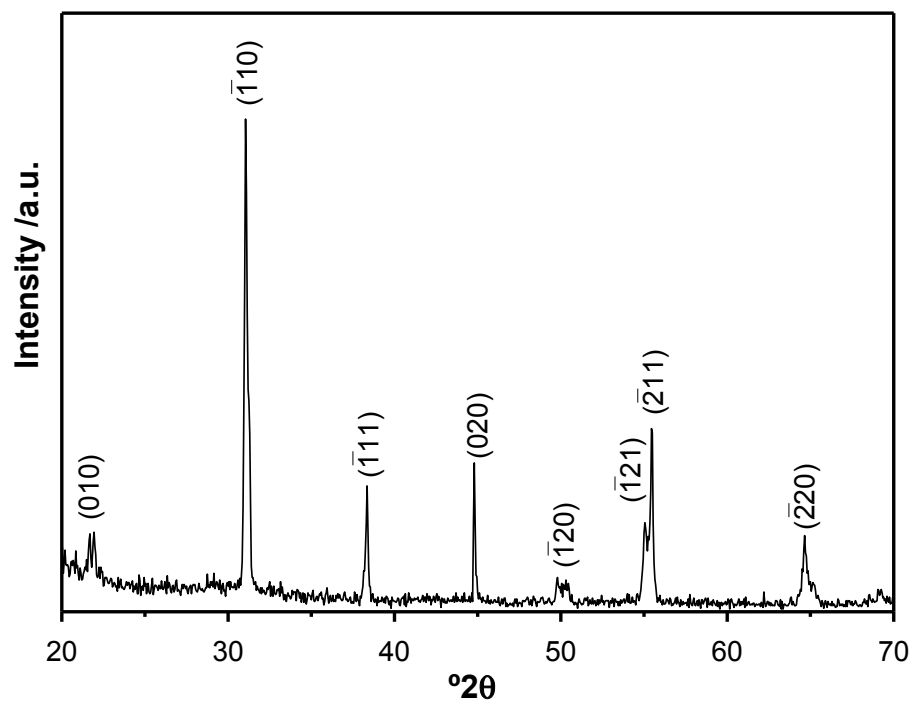

Figure 9. XRD pattern of the ceramic material.

To confirm these results, XRD of the powder obtained by grinding this piezoelectric ceramic material was done. Fig. 9 shows the diffraction pattern of this ceramic material. According to the JCPDS-ICDD patterns, all peaks observed were assigned to rhombohedral lead zirconium titanium oxide with a perovskite structure (JCPDS-ICDD 73-2022). The crystallographic planes of this perovskite structure are indicated in Fig. 9. Therefore, single phase of PZT is observed, which means that the piezoelectric material has a PZT based composition. The composition of this rhombohedral phase corresponds to a phase with higher content in zirconium than in titanium and confirms the results obtained by SEM/EDX.

At this point, students have analyzed the relation between crystallographic planes of a perovskite-type structure and its diffraction pattern.

Finally, layer III was also characterized by SEM, Fig. 10(a). This layer is formed by highly-sinterized irregular grains which correspond to metallic silver, as observed in EDX results.

In order to explain students how to recognize different types of microstructures, an interface region between PZT and silver was also analyzed, Fig. 10 (b). On the left zone of the image, PZT can be observed, whilst the right part is metallic silver.
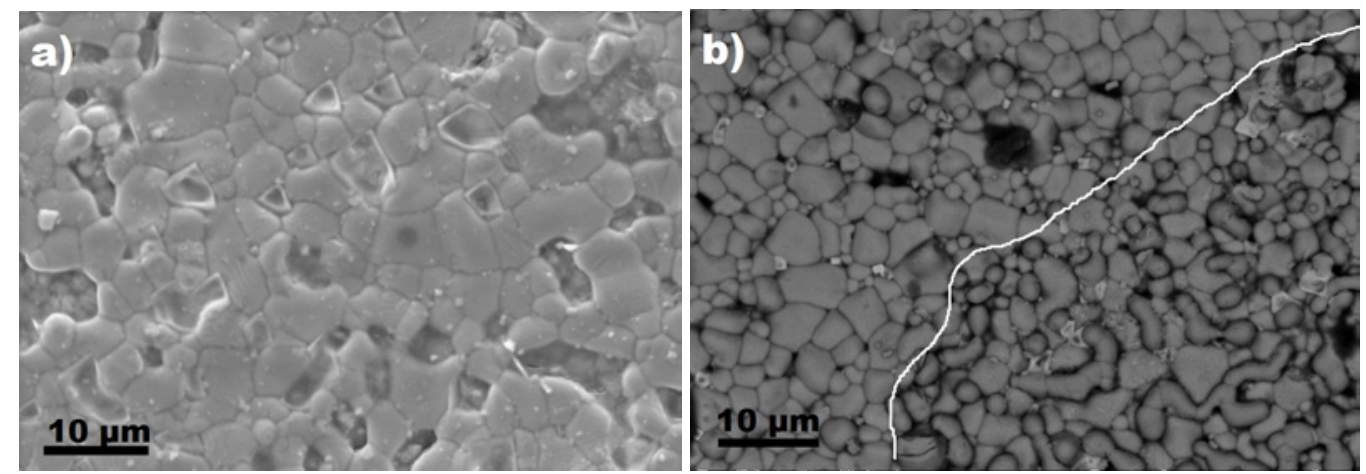

Figure 10. (a) SEM image of Layer III, (b) SEM image of the piezoelectric-silver interface.

Using the results obtained so far, students should be able to design a scheme of the components of the speaker, showing that the main component of the device is the piezoelectric material and it is the responsible of the acoustic response.

From all the results obtained in this work, a cross section image could be drawn, indicating the different materials present in a speaker, Fig 11. 


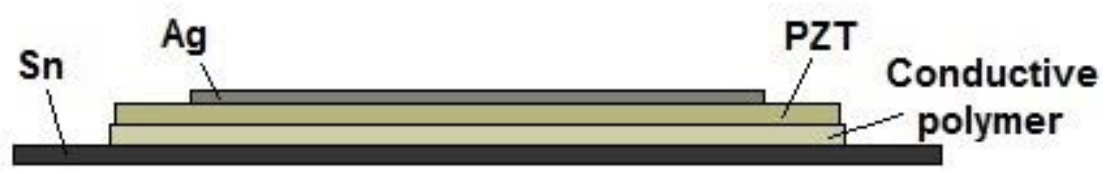

Figure 11. Cross-section image of a speaker

By following the steps of this work, students are able to determine the different types of materials (metals and ceramics) contained in the speaker and to understand in detail how it works, relating structure, composition and properties of each material.

\section{CONCLUSIONS}

PBL methodology used in this work represents an improvement in the content of the knowledge while simultaneously, it fosters the development of communication, problem-solving and self-directed learning skills.

Scanning Electronic Microscopy and X-ray Diffraction were used in this work to identify the piezoelectric material contained in a speaker. Crystalline phase information of the piezoelectric material was provided by $\mathrm{XRD}$, while the information of the chemical composition and morphology of the material was provided by SEM. XRD analysis showed that PZT was a single phase. Finally, the discovery of the presence of this type of material in this simple device offers a possibility to motivate students to learn these sciences, and allow them to know the importance of the Solid State Chemistry and the Materials Science in our society.

Students have achieved a better comprehension about how a speaker functions, working together disassembling a speaker of a musical birthday card, analyzing its components, searching information and characterizing the most important part of this device, the piezoelectric PZT material.

\section{ACKNOWLEDGMENTS}

We would like to thank the "Máster en Química Aplicada y Farmacológica" of the Universitat Jaume I, Castellón.

\section{REFERENCES}

[1] Amador, J.A.; Miles, L.; Peters, C.B., The Practice of Problem-Based Learning: A Guide to Implementing PBL in the College Classroom. Jossey Bass, 2006.

[2] Barr, R.D.; Tagg, J., "From teaching to learning - a new paradigm for undergraduate education", pp. 13-25, 1995.

[3] Bouhuijs, P.A.J., "Implementing problem based learning: Why is it so hard? REDU: Revista de Docencia Universitaria", 9(1), 2011.

[4] Duch, B.J.; Groh, S.E.; Allen, D.E. The Power of Problem-Based Learning. Sterling Stylus, 2001.

[5] Anthony R. West. Basic Solid State Chemistry. John Wiley and Sons. $2^{\text {nd }}$ Edition, 1999.

[6] A.M. Collieu, Derek J. Powney. Propiedades mecánicas y térmicas de los materiales. Ed. Reverte, 1977.

[7] Phase diagram of $\mathrm{PbTiO}_{3}-\mathrm{PbZrO}_{3}$ system. http://www.doitpoms.ac.uk/tlplib/piezoelectrics/pzt.php

[8] Tong, S.; Von Schirnding, Y.E.; Prapamontol, T., "Environmental lead exposure: a public health problem of global dimensions", Bulletin of the World Health Organization, 78 (9), 2000.

[9] López R. Síntesis y Caracterización de Materiales Piezoeléctricos Libres de Plomo. Universidad Nacional Autónoma de México. PhD Thesis, 2011.

[10] Beltrán Mir, H.; Sorlí Moliner, S.; Prades Tena, M.; Cordoncillo Cordoncillo, E. Práctica de Laboratorio de Ciencia de los Materiales, Publicación 359, Departamento de Química Inorgánica y Orgánica, Universidad Jaume I. Castellón, 2012. 
[11] Skoog, D.A.; Holler, F.J.; Crouch, S.R., Principles of instrumental analysis. Thomson Brooks/Cole. 6th Edition, 2007. 